\title{
ÁLCOOL COMO MEDIADOR SOCIAL EM UNIVERSITÁRIOS
}

\author{
Alcohol as a social mediator among university students
}

Alcohol como mediador social de universitarios

Artigo Original

\section{RESUMO}

Objetivo: Identificar a prevalência do consumo de álcool em estudantes do curso de Psicologia de uma instituição de ensino superior da cidade de Montes Claros-MG. Métodos: Pesquisa quantitativa, do tipo transversal e descritiva, realizada entre os meses de setembro e outubro de 2014. A população foi constituída por 116 universitários do curso de Psicologia da cidade de Montes Claros-MG. Para a coleta de dados, foram utilizados o Teste de Identificação de Desordens Devido ao Álcool (AUDIT), o Inventário de Expectativas e Crenças Pessoais Acerca do Álcool (IECPA), o Teste de Triagem do Envolvimento com Álcool, Cigarro e Outras Substâncias (ASSIST) e a Escala de Satisfação com o Suporte Social (ESSS). Para a análise descritiva dos dados, aplicou-se o programa SPSS, versão 19.0. Resultados: A amostra apresentou predominância do sexo feminino $(82,75 \%, n=96)$, pardos $(65,51 \%, n=76)$ e solteiros $(60,34 \%, n=70)$. Com relação à classificação de risco do AUDIT, constatou-se que 49,13\% ( $\mathrm{n}=57)$ dos participantes da pesquisa se enquadraram no nível 4, sendo considerados dependentes do álcool. Relataram fazer uso ocasional de fumo, álcool e outras substâncias, sendo uma classificação de nível 1 do ASSIT, com 94,82\% $(n=110)$. Sobre o IECPA, 87,06\% $(\mathrm{n}=101)$ dos avaliados se classificaram em nível 1, tendo baixa vulnerabilidade para os efeitos do álcool. Tratando-se do ESSS, 68,10\% $(\mathrm{n}=79)$ dos universitários apresentaram alto suporte social. Conclusão: Com relação à amostra pesquisada, constatou-se alta prevalência com dependência do álcool e outras drogas, lícitas e ilícitas.

Descritores: Consumo de Bebidas Alcoólicas; Estudantes; Psicologia.

\section{ABSTRACT}

Objective: To identify the prevalence of alcohol consumption in Psychology students of a higher education institution in the city of Montes Claros, MG. Methods: Quantitative crosssectional descriptive research conducted from September to October 2014. The population consisted of 116 Psychology students from the city of Montes Claros, MG. Data were collected using the Alcohol Use Disorders Identification Test (AUDIT), the Inventário de Expectativas e Crenças Pessoais Acerca do Álcool-IECPA (Inventory of Expectations and Personal Beliefs about Alcohol), the Alcohol, Smoking and Substance Involvement Screening Test (ASSIST) and the Escala de Satisfação com o Suporte Social - ESSS (Social Support Satisfaction Scale). Descriptive analysis of data was performed using SPSS 19.0. Results: The sample had a predominance of female gender $(82.75 \%, n=96)$, pardos $(65.51 \%, n=76)$ and single $(60.34 \%, n=70)$ individuals. Regarding the AUDIT risk classification, it was found that $49.13 \%(n=57)$ of the respondents were in the level 4 , considered alcohol dependence. They reported occasional use of alcohol, smoking and other substances, which refer to ASSIST level 1 classification, with $94.82 \%(n=110)$. Regarding the IECPA, 87.06\% $(n=101)$ of the individuals were classified as level 1, with low vulnerability to the effects of alcohol. As to the ESSS, $68.10 \%(n=79)$ of the students showed high social support. Conclusion: Regarding the sample studied, it was found a high prevalence of dependence on alcohol and other legal and illegal drugs.

Descriptors: Alcohol Consumption; Students; Psychology.

Recebido em: 16/11/2014 Revisado em: 20/03/2015 Aceito em: 28/06/2015

1) Universidade Estadual de Montes Claros - UNIMONTES - Montes Claros (MG) Brasil.

2) Faculdades Integradas do Norte de Minas - FUNORTE/SOEBRAS - Montes Claros (MG) - Brasil.

3) Centro de Pesquisa da Associação Educativa do Brasil - SOEBRAS - Montes Claros (MG) - Brasil. 


\section{RESUMEN}

Objetivo: Identificar la prevalencia del consumo de alcohol de estudiantes del curso de Psicología de una institución de enseñanza superior de la ciudad de Montes Claros-MG. Métodos: Investigación cuantitativa del tipo transversal y descriptiva realizada entre los meses de septiembre y octubre de 2014. La población se constituyó de 116 universitarios del curso de Psicología de la ciudad de Montes Claros-MG. Para la recogida de datos fueron utilizados el Teste de Identificación de Desordenes Debido al Alcohol (AUDIT), el Inventario de Expectativas y Creencias Personales Sobre el Alcohol (IECPA), el Teste de Selección del Envolvimiento con el Alcohol, Tabaco y Otras Sustancias (ASSIST) y la Escala de Satisfacción con el Apoyo Social (ESAS). Para el análisis descriptivo de los datos, se aplicó el programa SPSS, versión 19.0. Resultados: La muestra presentó la predominancia para el sexo femenino $(82,75 \%, n=96)$, pardos $(65,51 \%, n=76)$ y solteros $(60,34 \%, n=70)$. Respecto la clasificación de riesgo del AUDIT se constató que el 49,13\% ( $n=$ 57) de los participantes de la investigación se encuadraron en el nivel 4, siendo considerados dependientes del alcohol. Los mismos relataron el uso ocasional del tabaco, alcohol y otras sustancias con la clasificación del nivel 1 del ASSIT, con el 94,82\% ( $n=$ 110). Respecto el IECPA, el 87,06\% $(n=101)$ de los evaluados se clasificaron en nivel 1 con baja vulnerabilidad para los efectos del alcohol. Sobre el ESAS, el 68,10\% (n=79) de los universitarios presentaron elevado apoyo social. Conclusión: De la muestra investigada se constató elevada prevalencia de dependencia del alcohol y otras drogas lícitas e ilícitas.

Descriptores: Consumo de Bebidas Alcohólicas; Estudiantes; Psicología.

\section{INTRODUÇÃO}

Substâncias psicoativas (SPAs) são aquelas capazes de alterar o humor, o nível de percepção ou o funcionamento do sistema nervoso central. Tabaco, medicamentos e álcool são exemplos dessas substâncias, que, apesar de legalmente permitidas, causam diversos problemas sociais ${ }^{(1)}$.

Nessa perspectiva, o álcool é, hoje, uma das SPAs mais utilizadas pela população, sendo amplamente aceito. O seu uso como facilitador social traz muitas consequências: problemas de saúde, tanto física como psicológica; problemas nas relações familiares, sociais e de emprego; acidentes domésticos, do trabalho e de trânsito; comportamento violento e automutilação. Também está relacionado ao desenvolvimento de epidemias, como acidente vascular cerebral, câncer, absentismo escolar, problemas interpessoais e comportamentos sexuais de risco. É, ainda, um fator de risco na gravidez, estado nutricional e dieta. Vários estudos têm demonstrado a responsabilidade do consumo excessivo de álcool pelo insucesso entre estudantes universitários ${ }^{(2)}$.
O consumo de álcool, há séculos, acompanha a humanidade como um hábito lícito e socialmente aceitável, ligado à gratificação imediata, ao relaxamento e à facilitação da sociabilidade ${ }^{(3)}$. As diversas variáveis relacionadas a esse consumo e à dependência de bebidas alcoólicas apresentam associações bastante complexas. Fatores como escolaridade, renda e ocupação apresentam associações variadas com o uso de bebidas alcoólicas ${ }^{(4)}$.

O álcool é associado a alterações nos hábitos alimentares e estado nutricional do usuário, principalmente por afetar o apetite, a ingestão dos alimentos e/ou por agir diretamente sobre o metabolismo de alguns nutrientes específicos, como na absorção de vitaminas A e E, e minerais como cobre, zinco e selênio ${ }^{(5)}$. Dessa maneira, o usuário com dependência do álcool acaba por deixar de se alimentar, podendo vir a apresentar pelagra, um tipo de carência nutricional por falta de niacina, geralmente combinada à desnutrição energético-proteica, que ocorre com frequência devido à ingestão abusiva de bebidas alcoólicas ${ }^{(6)}$. Pesquisas apontam que o consumo de álcool contribui para mais de $10 \%$ dos problemas de saúde no Brasil, e seu alto consumo está relacionado ao excesso de peso. Muitos indivíduos dependentes de álcool apresentam sobrepeso, obesidade e circunferência da cintura acima dos padrões esperados ${ }^{(7)}$.

O consumo de substâncias alcoólicas entre os jovens é um fenômeno que tem causado problemas sociais, psicológicos e de saúde. Isso tem gerado uma questão de saúde pública, pois o uso excessivo de álcool tem alcançado estudantes dos mais variados graus educacionais ${ }^{(8)}$.

Os universitários da área da saúde constituem alvo de especial atenção no que se refere ao consumo de SPA. Nesse contexto, os universitários do curso de Psicologia, que se inserem entre as áreas da saúde e das ciências humanas, devem ter atenção privilegiada, pois, ao exercerem sua profissão, estarão diretamente envolvidos na identificação, prevenção e tratamento de indivíduos com problemas relacionados ao uso de $\mathrm{SPAs}^{(9)}$.

Existe na atualidade uma atenção especial a esse uso entre os universitários. O maior uso de álcool por essa população específica está relacionado a determinados fatores já identificados, como não possuir religião ou não frequentar celebrações religiosas, morar longe dos pais, e apresentar mais horas livres nos dias úteis e alta renda familiar. $\mathrm{O}$ ingresso na universidade pode se tornar um período crítico, pois a autonomia que se insere na vida de muitos estudantes é fator gerador de insegurança e de maior vulnerabilidade para o início e a manutenção do uso $\mathrm{SPA}^{(9)}$. Além disso, o uso cada vez mais precoce do consumo do álcool entre os jovens está associado a um maior risco de eles se tornarem dependentes ${ }^{(10)}$.

Diante disso, esta investigação se justifica por haver evidências de que há um alto consumo de bebidas 
alcoólicas entre os acadêmicos dos cursos da área da saúde. Sendo assim, há necessidade de verificar a real situação da utilização de álcool pelos acadêmicos de Psicologia de uma instituição de ensino superior da cidade de Montes ClarosMG e, através dos resultados apresentados, utilizá-los para a produção de estratégias, visando controlar o consumo excessivo de bebidas alcoólicas.

\section{MÉTODOS}

Trata-se de uma pesquisa de caráter descritivo, com abordagem quantitativa e de corte transversal.

Aamostra, do tipo não probabilística e por conveniência, foi constituída de 116 estudantes na faixa etária entre $19 \mathrm{e}$ 47 anos $(26,2 \pm 5,7)$, matriculados e frequentes no curso de Psicologia de uma instituição privada de ensino superior da cidade de Montes Claros-MG. Após a autorização da coordenação do curso para a realização da pesquisa, fez-se uma reunião com os possíveis participantes da pesquisa, e todos que aceitaram participar de forma voluntária do estudo assinaram o termo de consentimento livre e esclarecido (TCLE).

Após isso, aplicou-se o instrumento de coleta dos dados pelas próprias pesquisadoras nos meses de setembro e outubro 2014, sempre nos horários de aulas.

Para a coleta dos dados, utilizou-se um questionário sociodemográfico para caracterização do grupo, contendo dados descritivos (sexo, etnia, estado civil e o período cursado) com a finalidade de caracterizar o grupo amostral.

Além disso, aplicaram-se quatro questionários específicos. O primeiro foi o Teste para Identificação de Problemas Relacionados ao Uso de Álcool (AUDIT), composto por 10 questões que permitem respostas com pesos pré-estabelecidos de 0 a 4 . Ao final, é somada a pontuação, sendo verificadas as "zonas de risco": zona I (pontuação de 0 a 7 - padrão de baixo risco; indicação de tratamento: educação sobre o álcool), zona II (pontuação de 8 a 15 - padrão de médio risco; indicação de tratamento: conselho simples), zona III (pontuação de 16 a 19 - padrão de alto risco; indicação de tratamento: conselho simples, com terapia breve e acompanhamento continuado), zona IV (pontuação igual ou superior a 20 - prováveis portadores de síndrome de dependência do álcool). Esse instrumento é o primeiro de rastreamento específico, criado pela Organização Mundial de Saúde (OMS) para identificar pessoas com consumo nocivo do álcool, como também aquelas que apresentam dependência de álcool, nos últimos 12 meses $^{(10)}$.

Na sequência, utilizou-se o Inventário de Expectativas e Crenças Pessoais acerca do álcool (IECPA), que visa conhecer o que as pessoas pensam sobre os efeitos do álcool. Consiste em um instrumento com 61 itens, cada qual contendo cinco alternativas de resposta tipo likert com escores de 1 a 5 . Em seguida, o Teste de Triagem do Envolvimento com Álcool, Cigarro e Outras Substâncias (ASSIST), que consiste em um instrumento autoaplicável, em formato de questionário, produzido pela Organização Mundial de Saúde (OMS), contendo oito questões sobre o uso de nove classes de substâncias psicoativas (tabaco, álcool, maconha, cocaína, estimulantes, sedativos, inalantes, alucinógenos e opiáceos). Cada resposta corresponde a um escore, que varia de 0 a 4 , e a soma total pode variar de 0 a 20. Considera-se a faixa de escore de 0 a 3 como indicativa de uso ocasional, de 4 a 15 como indicativa de abuso, e $\geq 16$ como sugestiva de dependência.

Por último, aplicou-se a Escala de Satisfação com o Suporte Social (ESSS), instrumento para avaliar um conjunto de medidas que expressam saúde, bem-estar ou mal-estar, ou que estão intimamente ligadas a essas variáveis. Consiste numa escala de autopreenchimento, composta por 15 frases que permitem ao avaliado assinalar o seu grau de concordância com cada uma delas (se a frase se aplica ou não à sua situação individual), seguindo uma escala de tipo likert, com 5 posições de resposta. A ESSS possibilita entender quatro fatores e dimensões: satisfação com amigos/amizades; intimidade; satisfação com a família; e atividades sociais ${ }^{(11)}$.

Os dados foram analisados de forma descritiva, com valores em porcentagem, média e desvio padrão, pelo programa SPSS, versão 19.0.

$\mathrm{O}$ presente estudo foi submetido e aprovado pelo Comitê de Ética em Pesquisa (CEP) da Associação Educativa do Brasil - SOEBRAS, sob o parecer n ${ }^{\circ} 530.651$.

\section{RESULTADOS}

A maioria da amostra era do sexo feminino $(82,75 \%$, $\mathrm{n}=96)$, de etnia parda $(65,51 \%, \mathrm{n}=76)$, solteira $(60,34 \%$, $\mathrm{n}=70)$ e cursando $6^{\circ}$ período $(36,20 \%, \mathrm{n}=42)$ (Tabela I).

Com relação aos resultados obtidos por meio do teste aplicado para a Identificação de Problemas Relacionados ao Uso de Álcool (AUDIT), verificou-se que a maioria dos alunos $(49,13 \%, n=57)$ se encontrava na zona de risco IV - dependência do álcool. Sobre a frequência do uso do cigarro e outras substancias psicoativas nas mesmas situações em que o álcool é consumido (ASSIST), foi verificado que $94,82 \%(\mathrm{n}=110)$ dos universitários fazem uso ocasional de algum tipo de substância. Ao avaliar as expectativas pessoais acerca dos efeitos positivos do uso do álcool e a vulnerabilidade ao alcoolismo dos acadêmicos entrevistados, verificou-se que $87,6 \%(n=101)$ apresentavam baixa expectativa/baixa vulnerabilidade ao alcoolismo. O ESSS analisa a relação entre o álcool e a socialização, na qual se encontrou que $68,10 \%(n=79)$ dos universitários fazem uso da bebida alcoólica como suporte social (Tabela II). 
Tabela I - Caracterização dos estudantes do curso de Psicologia de uma instituição privada de ensino superior. Montes Claros-MG, 2014. $(\mathrm{n}=116)$.

\begin{tabular}{lccc}
\hline Variável & & $\mathbf{n}$ & $\mathbf{\%}$ \\
\hline \multirow{2}{*}{ Sexo } & Masculino & 20 & 17,25 \\
& Feminino & 96 & 82,75 \\
& Asiático & 0 & 0 \\
Etnia & Branco & 27 & 23,30 \\
& Índio & 04 & 3,44 \\
& Negro & 09 & 7,75 \\
& Pardo & 76 & 65,51 \\
\multirow{4}{*}{ Estado civil } & Solteiro & 70 & 60,34 \\
& Casado & 23 & 19,84 \\
& Separado & 07 & 6,03 \\
& Viúvo & 0 & 0 \\
& Namoro & 16 & 13,79 \\
& $5^{\circ}$ & 11 & 9,48 \\
& $6^{\circ}$ & 42 & 36,22 \\
& $7^{\circ}$ & 04 & 3,44 \\
& $8^{\circ}$ & 18 & 15,51 \\
& $9^{\circ}$ & 13 & 11,22 \\
& $10^{\circ}$ & 28 & 24,13 \\
\hline
\end{tabular}

Tabela II - Análise da prevalência do consumo de álcool dos estudantes do curso de Psicologia de uma instituição privada de ensino superior. Montes Claros-MG, 2014. ( $\mathrm{n}=116)$.

\begin{tabular}{lcccc}
\hline QUESTIONÁRIO & NÍVEL & n & \% & CLASSIFICAÇÃO \\
\hline \multirow{3}{*}{ AUDIT } & 1 & 44 & 37,93 & Baixo Risco \\
& 2 & 13 & 11,20 & Médio Risco \\
& 3 & 02 & 1,72 & Alto Risco \\
\multirow{2}{*}{ ASSIST } & 4 & 57 & 49,13 & Dependência Álcool \\
& 1 & 110 & 94,82 & Uso Ocasional \\
IECPA & 2 & 06 & 5,17 & Uso Abusivo \\
& 1 & 101 & 87,06 & Baixa Expectativa - Baixa Vulnerabilidade \\
& 2 & 15 & 12,93 & Alta Expectativa - Alta Vulnerabilidade \\
ESSS & 1 & 02 & 1,72 & Baixo Suporte Social \\
& 2 & 35 & 30,17 & Médio Suporte Social \\
& 3 & 79 & 68,10 & Alto Suporte Social \\
\hline
\end{tabular}

\section{DISCUSSÃO}

Esta pesquisa avaliou a prevalência do consumo de álcool entre os acadêmicos do curso de Psicologia de uma instituição privada de ensino superior da cidade de Montes Claros-MG.
Com relação ao grupo amostral, houve um predomínio do sexo feminino. Em concordância com os resultados do presente estudo, um estudo anterior ${ }^{(12)}$ que investigou o consumo de álcool verificou que maioria era do sexo feminino $(61,6 \%)$ e apenas $38,4 \%$ da amostra era do sexo 
masculino, assim como em vários outros trabalhos sobre o uso do álcool, o que corrobora com os achados ${ }^{(13-16)}$.

Quanto à etnia, a maioria dos acadêmicos avaliados se considerava parda. Em contrapartida, um estudo ${ }^{(15)}$ realizado analisando a relação entre a etnia de universitários e o uso de álcool, tabaco e drogas entre universitários demonstrou que a maioria $(65,8 \%)$ dos universitários se considerava branca, $20,3 \%$ eram pardos, seguidos dos negros $(6,3 \%)$, asiáticos $(2,5 \%)$ e índios $(0,5 \%)$.

$\mathrm{O}$ estado civil de maior prevalência foi solteiro. Os resultados obtidos por um estudo realizado na cidade de Montes Claros ${ }^{(13)}$, localizada no norte do estado de Minas Gerais, condiz com os resultados do estudo em questão, no qual a maioria dos acadêmicos é solteira. Isso pode justificar o alto consumo de bebidas alcoólicas, pois os solteiros possuem vida social mais ativa do que os casados e os que namoram.

A pesquisa foi realizada entre os acadêmicos do $5^{\circ}$ ao $10^{\circ}$ período do curso de Psicologia, com predomínio de acadêmicos do $6^{\circ}$ período. Por outro lado, outro estudo mostrou que a maioria dos acadêmicos participantes da pesquisa era do $2^{\circ}$ período $(21 \%)$, seguido do $3^{\circ}$ período $(19 \%)^{(15)}$.

Em consonância com os resultados da presente pesquisa, um estudo ${ }^{(17)} \mathrm{em}$ duas faculdades públicas de Alagoas relatou que a maioria dos estudantes participantes é do primeiro período (24,2\%), seguido daqueles que estão cursando o terceiro período (23,3\%). Acredita-se que esses resultados se justificam pelo fato de que, nos semestres iniciais, as salas possuem maior número de alunos.

Com relação aos resultados obtidos por meio do AUDIT, verificou-se que a maioria dos avaliados se encontra na zona de risco IV (dependência do álcool). O consumo elevado de bebidas alcoólicas tem se tornado um grande problema de saúde pública no Brasil, uma vez que os adolescentes e jovens adultos iniciam o uso dessas substâncias precocemente. À medida que vai aumentando o número de pessoas que consomem bebidas alcoólicas, maiores são as ocorrências negativas, tanto psicológicas como sociais ${ }^{(18,9)}$.

Um estudo realizado com 174 alunos de uma universidade pública de São Luís-MA observou que 59\% dos participantes responderam já ter consumido de forma abusiva algum tipo de bebida alcoólica, dos quais 20,7\% relataram já ter se embriagado nos últimos 19 dias, e 14,9\%, nos últimos cinco dias que antecedem a coleta ${ }^{(19)}$.

Pode-se observar que existe um número elevado de pessoas que fazem uso abusivo de bebidas alcoólicas, apresentando vulnerabilidade à dependência alcoólica. Aqueles indivíduos que se enquadram na zona de risco IV do questionário AUDIT são pessoas que necessitam de uma avaliação especializada para diagnosticar a dependência alcoólica e um tratamento especifico para combater esse mal.

Em contrapartida, um estudo realizado na cidade de Piracicaba, interior do estado de São Paulo, avaliou o consumo do álcool e a qualidade de vida em universitários, sendo sua amostra composta por 170 indivíduos de ambos os sexos. Nele, observou-se que $68,20 \%$ dos participantes faziam uso de baixo risco do álcool, 21,80\% faziam uso de alto risco, e 5,29\% dos alunos se encontravam em provável dependência do álcool ${ }^{(20)}$.

Do mesmo modo, resultados encontrados por uma pesquisa realizada em uma escola pública em São Paulo com 112 alunos do curso de Enfermagem, com idades entre 17 e 28 anos, verificaram que $79,5 \%$ dos indivíduos se encontravam com baixo risco de dependência do álcool ${ }^{(14)}$.

Outro estudo, feito em um Hospital de Clinicas da Universidade Estadual de Campinas com uma amostra composta por 4.352 indivíduos com idade acima dos 18 anos, mostrou que $9,7 \%$ dos entrevistados fazem uso de alto risco do álcool. Acredita-se que essa porcentagem é baixa ao comparar o número de pessoas participantes, porém, a maioria desses indivíduos é jovem e, consequentemente, mais vulnerável ao consumo do álcool ${ }^{(15)}$.

Apesar de os resultados da presente pesquisa serem contraditórios, leva-se em consideração que os resultados dos estudos citados são animadores, mas não diminui as chances de essas porcentagens de indivíduos em consumo de risco virem a aumentar, fazendo-se necessário campanhas de conscientização aos riscos ocasionados pelo uso do álcool.

Sobre a questão da utilização do uso de cigarro e outras substâncias psicoativas nas mesmas situações em que o álcool é consumido, foi verificado que a maioria dos universitários faz uso ocasional de algum tipo de substância, ou seja, os indivíduos utilizam o álcool e o cigarro nas mesmas circunstâncias, porém, apresentam baixo risco de se tornarem dependentes da nicotina.

Em concordância com o presente achado, um estudo feito no Centro de Ciências Humanas e Naturais da UFES com 300 alunos participantes do curso de Psicologia observou que as substâncias mais usadas pelos universitários são o álcool $(85,07 \%)$ e o tabaco $(33,03 \%)^{(9)}$.

Apesar de esses indivíduos possuírem alto contato com outras substancias psicoativas, pode-se observar que, entre essas drogas, a preferência dos jovens é o cigarro, ressaltando que este, na maioria das vezes, está presente junto com o álcool. Essa afirmativa corrobora com os achados da presente pesquisa.

O tabaco e o álcool são substâncias prejudiciais à saúde, e até 2020 serão fatores determinantes de mortes e incapacidade de indivíduos predisposto à dependência ${ }^{(21)}$. 
Cerca de $84 \%$ fazem uso eventual do álcool, porém, qualquer quantidade consumida pode acarretar danos para o próprio individuo e até mesmo para pessoas ao seu redor ${ }^{(22)}$.

O consumo do álcool cada vez mais crescente pode levar as pessoas a utilizarem outros tipos de substâncias psicoativas. Estudo relata, ainda, que o uso do tabaco é a segunda droga mais consumida entre os jovens no mundo, e que existe uma relação entre as duas substâncias, pois aqueles que fazem uso do álcool utilizam ou já utilizaram o tabaco ${ }^{(22)}$. Na presente pesquisa, os avaliados também apresentaram baixa expectativa/baixa vulnerabilidade ao alcoolismo.

Achados no $1^{\circ}$ Levantamento Nacional sobre o Uso do Álcool, Tabaco e outras Drogas entre Universitários mostram que $22 \%$ dos acadêmicos participantes estavam em risco de desenvolver dependência do álcool e com uma provável motivação para a utilização indevida de bebidas alcoólicas ${ }^{(16)}$.

Pesquisa realizada na Universidade Federal do Rio Grande mostra que, quanto às consequências do álcool nas vidas desses indivíduos, $23,7 \%$ responderam sentir tonturas e houve até casos de quedas, e $3 \%$ dos indivíduos relataram a ocorrência de coma alcoólico ${ }^{(23)}$.

Para verificar a relação entre o álcool e a socialização, utilizamos o questionário ESSS (Escala de Satisfação com o Suporte Social), no qual se encontrou que $68,10 \%$ dos universitários fazem uso da bebida alcoólica a fim de ter algum tipo de suporte social.

Dentro desse contexto, um estudo feito na Universidade Federal de Uberlândia mostrou que 66,3\% dos universitários consumiam bebidas alcoólicas e $65,17 \%$ bebiam ocasionalmente ${ }^{(24)}$. O provável motivo do elevado consumo de álcool é a sua grande aceitação social. O autor afirma, ainda, que é mais comum os pais desses indivíduos alertarem o filho contra o uso de um cigarro de maconha do que com aquele que bebe todos os dias.

Propagandas de bebidas alcoólicas influenciam o uso dessas substâncias pelos jovens, uma vez que elas, na maioria das vezes, são voltadas para esse público. No Brasil, quase não há programas de alerta aos perigos do abuso do álcool, e as propagandas sobre bebidas alcoólicas quase não possuem restrições ao serem veiculadas ao público ${ }^{(25)}$.

Muitos desses indivíduos não possuem conhecimentos do que tais substâncias podem acarretar para saúde. Quando o indivíduo se torna dependente dessas substâncias, a retirada total ou até mesmo parcial desse mal se torna algo mais difícil, pois indivíduos dependentes rejeitam tratamentos e ajudas para abandonar o vício, o que torna isso um grande desafio para quem luta contra as drogas e o álcool.
A amostra pequena e a seleção de apenas um curso se apresentam como limitações deste estudo. Assim, considerase importante a ampliação de estudos sobre os riscos causados pelo uso abusivo do álcool entre universitários da área da saúde, sendo estes detentores do conhecimento e promotores da saúde.

\section{CONCLUSÃO}

Com relação à amostra pesquisada, constatou-se alta prevalência em dependência do álcool e outras drogas lícitas e ilícitas.

\section{REFERÊNCIAS}

1. Zeitoune RCG, Domingos AM, Ferreira VS, Maia AC, Silveira HS. O conhecimento de adolescentes sobre drogas lícitas e ilícitas: uma contribuição para a enfermagem comunitária. Esc Anna Nery Rev Enferm. 2012;16(1):57-63.

2. Tovar LLA, Caballero AJD, Martinez FDG, Pallares MAS. Consumo de bebidas alcoólicas e fatores relacionados em estudantes de odontologia. Rev Clin Med Fam. 2010;3 (2):93-8.

3. Barbor TF, Caetano R, Casswell S, Edwards G, Giesbrect N, Graham K, et al. Alcohol: no ordinary commodity: research and public policy. $2^{\text {nd }}$ ed. Oxford: Oxford University Press; 2010.

4. Ferreira LN, Bispo Júnior JP, Sales ZN, Casotti CA, Braga Junior ACR. Prevalência e fatores associados ao consumo abusivo e à dependência de álcool. Ciênc Saúde Coletiva. 2013;18(11):3409-18.

5. Schlichting S, Boog MCF, Campos CJG. Almoço como momento terapêutico: uma abordagem de educação em saúde com mulheres alcoolistas. Rev Latinoam Enferm. 2007;15(3):384-90.

6. Bastos FL, Bertoni N, Hacker MA. Consumo de álcool e drogas: principais achados de pesquisa de âmbito nacional, Brasil 2005. Rev Saúde Pública. 2008;42(Supl 1):109-17.

7. Kachani AT, Brasiliano S, Hochgraf PB. O impacto do consumo alcoólico no ganho de peso. Rev Psiquiatr Clín. 2008;35(Supl 1):21-4.

8. Agante DMC. Comportamentos relacionados com o consumo de bebidas alcoólicas durante as festas académicas nos estudantes do ensino superior [dissertação]. Coimbra: Universidade de Coimbra; 2009. 
9. Santos MVF, Pereira DS, Siqueira MM. Uso de álcool e tabaco entre estudantes de Psicologia da Universidade Federal do Espírito Santo. J Bras Psiquiatr. 2013;62(1):22-30.

10. Lourenço FCB. Consumo do álcool nos adolescentes numa escola secundária de Vale de Souza [monografia]. Porto: Universidade Fernando Pessoa; 2008.

11. Bezerra-Ribeiro, M. Percepção de suporte social e consumo de álcool em desempregados [dissertação]. São Bernardo do Campo: Universidade Metodista de São Paulo; 2007.

12. Ferreira APS. O consumo de álcool e comportamentos de risco nos estudantes do ensino superior [dissertação]. Aveiro: Universidade de Aveiro; 2008.

13. Rocha LA, Lopes ACFMM, Martelli DRB, Lima VB, Martelli-Júnior H. Consumo de Álcool entre Estudantes de Faculdades de Medicina de Minas Gerais, Brasil. Rev Bras Educ Méd. 2011;35(3):369-75.

14. Funai A, Pillon SC. Uso de bebidas alcoólicas e aspectos religiosos em estudantes de enfermagem. Rev Eletrônica Enferm. 2011;13(1):24-9.

15. Lima DD, Azevedo RCS, Gaspar KC, Silva VF, Mauro MLF, Botega NJ. Tentativa de suicídio entre pacientes com uso nocivo de bebidas alcoólicas internados em hospital geral. J Bras Psiquiatr. 2010;59(3):167-72.

16. Presidência da República (BR), Secretaria Nacional de Políticas sobre Drogas. I levantamento nacional sobre o uso de álcool, tabaco e outras drogas entre universitários das 27 capitais brasileiras. Brasília: SENAD; 2010.

17. Pedrosa AAS, Camacho LAB, Passos SRL, Oliveira RVC. Consumo de Álcool Entre Estudantes Universitários. Cad Saúde Pública. 2011;27(8):161121.

18. Anjos KF, Santos VC, Almeida OS. Caracterização do Consumo de Álcool entre Estudantes do Ensino Médio. Rev Baiana Saúde Pública. 2012;36(2):418-31.
19. Carvalho DA, Gomes RIB, Sousa VEC, Sardinha AHL, Costa Filho MR. Hábitos Alcoólicos Entre Universitários de uma Instituição Pública. Ciênc Cuid Saúde. 2011;10(3):571-7.

20. Manzatto L, Rocha TBX, Vilela Júnior GB, Lopes GM, Sousa JA. Consumo de álcool e qualidade de vida em Estudantes universitários. Rev Fac Educ Fis Unicamp. 2011;9(1):37-53.

21. Arnauts I, Oliveira MLF. Intoxicação alcoólica em crianças e adolescentes: dados de um centro de assistência toxicológica. Esc Anna Nery Rev Enferm. 2011;15(1):83-9.

22. Oliveira HF, Martins LC, Reato LFN, Akerman M. Fatores de risco para uso do tabaco em adolescentes de duas escolas do município de Santo André, São Paulo. Rev Paul Pediatr. 2010;28(2):200-7.

23. Baumgarten LZ, Gomes VLO, Fonseca AD. Consumo Alcoólico entre universitários(as) da área da Saúde da Universidade Federal do Rio Grande/RS: Subsídios Para Enfermagem. Esc Anna Nery Rev Enferm. 2012;16(3):530-5.

24. Paduani GF, Barbosa GA, Morais JCR, Pereira JCP, Almeida MF, Prado MM, et al. Consumo de álcool e fumo entre os estudantes da Faculdade de Medicina da Universidade Federal de Uberlândia. Rev Bras Educ Méd. 2008;32(1):66-75.

25. Faria R, Vendrame A, Silva R, Pinsky I. Propaganda de Álcool e Associação ao Consumo de Cerveja por Adolescentes. Rev Saúde Pública. 2010;45(3):441-7.

\section{Endereço para correspondência:}

Wellington Danilo Soares

Avenida Ruy Braga, s/n

Bairro: Vila Mauriceia

CEP: 39401-089 - Montes Claros - MG - Brasil

E-mail:wdansoa@yahoo.com.br 